Reprod. Nutr. Dévelop. 1980, 20 (2), 557-567.

\title{
Les gangues ovulaires des Amphibiens (protéines sécrétées par l'oviducte) et leurs rôles dans la fécondation.
}

\author{
par P. JÉGO, J. JOLY, C. BOISSEAU \\ Laboratoire de Biologie de la Reproduction. \\ Groupe de Recherches en Biologie Cellulaire (C.N.R.S. LA 256) \\ Université de Rennes, 35042 Rennes Cedex, France.
}

Summary. Amphibian jelly envelopes, proteins secrefed by the oviduct and surrounding the eggs : Their role in fertilization.

The jelly envelopes, surrounding amphibian eggs and playing a major part in the initial steps of fertilization, are deposided around the eggs as they traverse the different parts of the oviduct. These envelopes form 5 to 8 morphologically distinct layers according to the species, i.e. in Anura and Caudata they are organized differently, and are composed of about ten different macromolecular components (glycoproteins). The innermost layer is rich in sulfate and poor in fucose, a carbohydrate abundant in the other layers. This is due to a fucosyltransferase deficit in the cranial part of the oviduct secreting this layer.

The cranial and the medial oviduct secretory products show a lectin carbohydratelike reaction which is $\mathrm{pH}$-dependent and can be inhibited by carbohydrates like glucuronolactone. This reaction has made it possible to isolate, by affinity chromatography, the main secretory component of the cranial oviduct (Spop).

This paper discusses the main functions of the jelly layers of the egg, with particular reference to fertilization. The role of their macromolecular components in sperm capacitation is clear in Anura ; experimental investigation in Urodeles is more difficult. We propose several hypotheses to explain the molecular interactions leading to capacitation. Block to polyspermy in amphibians is discussed.

\section{Introduction.}

Depuis les origines de l'embryologie expérimentale, l'œuf des Amphibiens est l'un des plus utilisés pour étudier la fécondation (Spallanzani, 1785 ; Bataillon, 1919). Parmi les raisons qui justifient ce choix, on peut citer la relative facilité d'obtenir un matériel abondant durant une grande partie de l'année, la simplicité de sa manipulation liée à des dimensions accessibles (diamètre de l'ordre du millimètre) et sa survie sans problème dans l'eau douce. Même si des progrès essentiels ont été accomplis dans la dernière décennie sur d'autres types d'organismes comme les Echinodermes (Epel, 1978) ou les Mammifères (Thibault, 1975, 1976 ; Grobstein, 1979), l'ovocyłe des Amphibiens continue à susciter l'intérêt des expérimentateurs. La maturation et son 
déterminisme (Baulieu ef al., 1978), la fécondafion et ses mécanismes infimes ainsi que les premiers stades du développement ont donné lieu à un très grand nombre de travaux récents.

Dans le déroulement de la fécondation, les phases initiales jouent un rôle particulièrement important. Elles concernent essentiellement la traversée, par les spermatozoïdes, des gangues ou enveloppes ovulaires. On sait depuis longtemps (Aplington, 1957 ; Katagiri, 1966) que les ovocytes d'Amphibiens dépourvus de gangues ne peuvent être fécondés même s'ils sont mis au contact de nombreux spermatozoïdes. Shivers et James (1970a) ont proposé le terme de capacitation pour désigner les modifications subies au cours de la traversée de ces enveloppes par les spermatozoïdes fécondants, par analogie avec un phénomène bien connu chez les Mammifères. En effet, dans les deux cas, ces modifications se produisent au contact des produits de sécrétion des voies génitales femelles. Elles se traduisent essentiellement (Thibault, 1975) par une déstabilisation de la membrane plasmique ef de la membrane externe de l'acrosome. Elles aboutissent à la réaction acrosomienne. Les enzymes protéolytiques de l'acrosome sont ainsi mises à nu et permettent au spermatozoïde de poursuivre son parcours jusqu'au contact de l'ovocyte en traversant l'enveloppe vitelline (Katagiri, 1966, 1973 ; Mełz, 1967 ; Picheral, 1977). Il est donc très important d'étudier la structure ef la composition chimique des différentes gangues pour tenter de rechercher quels sont les constituants susceptibles d'intervenir dans ces phénomènes de capacitation et de mieux comprendre les phases précoces de la fécondation.

\section{Mise en place et structure des gangues ovulaires}

Les œufs d'Amphibiens sont recouverts, au cours de leur transit dans l'oviducte, du produit de sécrétion des segments successifs de ce canal qui forment les gangues ou capsules ovulaires. Au nombre de trois à huit selon les espèces, ces enveloppes sont d'épaisseur ef de consistance variées. En général des couches plus ou moins fluides ef visqueuses alternent avec des couches minces et plus résistantes. La ponte étant le plus souvent aquatique, les gangues gonflent rapidement au contact de l'eau et forment un ensemble de couches dont les rôles sont multiples et seront évoqués plus loin. En ce qui concerne les Urodèles, on peut prendre comme exemple l'œuf du Pleurodèle de Waltl. L'enveloppe vitelline (ou chorion), sécrétée dans le follicule ovarien, est une couche protéique mince, étroitement appliquée à la surface de l'œuf dont le diamètre atteint $1,6 \mathrm{~mm}$ environ. Ce dernier baigne dans la gangue interne (fig. 1) très fluide et peut s'orienter librement selon son gradient de densité. Cette première gangue est déposée lors du passage de l'œuf dans la partie craniale de l'oviducte (oviducte antérieur) qui fait suite à la trompe ciliée. Elle réagit fortement au bleu Alcian qui atteste la présence de mucopolysaccharides très acides. Elle est limitée extérieurement par une « copsule » mince et résistante, fortement APS positive, qui correspond à la gangue moyenne déposée au passage dans le segment moyen de l'oviducte. La chambre capsulaire ainsi formée a un diamètre de l'ordre de $3 \mathrm{~mm}$. La gangue externe, très épaisse (de l'ordre de $5 \mathrm{~mm}$ après hydratation) est déposée par le long oviducte postérieur ef se décompose en trois couches successives moins compactes que la précédente car plus hydratées el qui réagissent également à l'APS. Le diamètre de l'œuf 
hydraté entouré de ses cing gangues atteint environ $12 \mathrm{~mm}$. La gangue externe gluante peut fixer l'œuf au substratum.

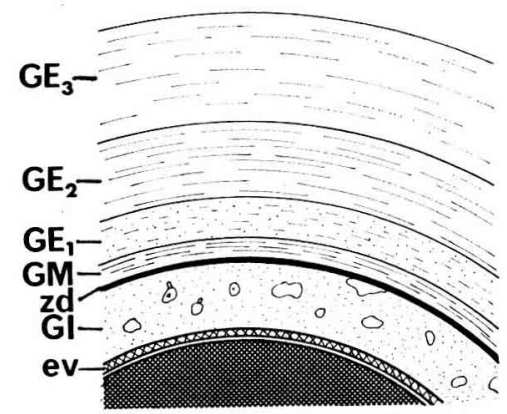

PLEURODELES

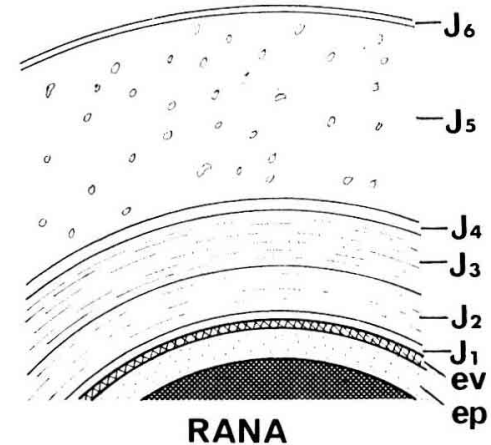

RANA

FIG. 1. - Représentation schématique des gangues ovulaires (sécrétées par l'oviducte) chez un Urodèle Pleurodeles waltl (d'après Boisseau et al., 1974) et chez un Anoure, Rana pipiens (d'après Shivers ef James, 1970b). Les schémas correspondent à des œufs fixés et déshydratés ; sur le matériel frais, les gangues, après hydratation, sont considérablement plus épaisses.

Nomenclature : - ev, enveloppe vitelline (chorion, enveloppe pellucide...) ; - pour Pleurodeles : GI, gangue interne; $G M$, gangue moyenne; $G E_{1}$ à $G E_{a}$, gangue externe, zd, zone densifiée (voir le texte) ; - pour Rana $J_{1}$ à $J_{6}$, couches successives de " gelée " (jelly) ; ep, espace périvitellin (apparaissant après la fécondation).

En ce qui concerne les Anoures, la plupart des espèces ne possèdent pas de chambre capsulaire. On peut prendre pour exemple l'œuf de Rana pipiens étudié par Shivers et James (1970b) (fig. 1). Contrairement au cas précédent, la gangue interne y est mince, peu fluide ef étroitement attachée à l'enveloppe vitelline. C'est d'ailleurs cette dernière qui se soulève de l'œuf et permet l'orientation précoce de l'embryon selon la force de gravité à l'intérieur de ses enveloppes. Cette gangue interne est entourée par deux couches moyennes que l'on distingue aisément à l'aide de réactions hislochimiques. Vient ensuite une gangue très hydratée qui représente à elle seule les trois quarts de l'épaisseur totale et qui est bordée extérieurement par une mince couche superficielle gluante. Les gangues des Amphibiens forment donc un ensemble complexe d'enveloppes dont la structure ef les propriétés sont, bien entendu, étroitement liées à leur constitution chimique (cf. Salthe, 1963 ; Joly, 1980).

\section{Composition chimique des gangues ovulaires}

Les gangues ovulaires des Amphibiens se caractérisent en premier lieu par le fait qu'elles renferment toutes des glucides dans des proportions assez élevées (30 à 50 p. 100 du poids sec) (Folkes et al., 1950 ; Minganti et d'Anna, 1957, 1958 ; Bolognani et al., 1966 ; Lee, 1967 ; Katagiri, 1973 ; Jégo, 1974 ; Yurewicz et al., 1975). Deux catégories de glucides sont très bien représentées : les oses neułres (galactose, fucose) ef les osamines (glucosamine ef galactosamine) qui sont en quantités sensiblement égales ef constituent à eux seuls au moins 90 p. 100 des glucides. Les acides sialiques sont beaucoup moins abondants que certains dosages (pas assez spécifiques) ne le 
laissaient supposer ; ils ne constituent en faif que 1 à 3 p. 100 du poids sec total des gangues (Bolognani et al., 1966 ; Katagiri, 1973 ; Jégo, 1974 ; Yurewicz et al., 1975 ; De Martinez ef al., 1975). L'absence d'acide uronique indique que ces produits ne sont sûrement pas des glycosamino-glycuronoglycanes. Même si la présence de quelques polyosides n'esł pas à exclure totalement, les gangues ovulaires sont essentiellement constituées de glycoproféines puisqu'il y a superposition entre les électrophorégrammes colorés par des techniques mettant en évidence les protéines (bleu de Coomassie ou noir amide) et ceux colorés par l'APS, spécifique des glucides.

La fraction protéique de ces glycoprotéines se caractérise par une forte teneur en acides aminés alcool (sérine et thréonine) (Oliphant et Hedrick, 1971 ; Jégo, 1976) ce qui différencie très netfement les produits de sécrétion de l'oviducte des Amphibiens de ceux des Oiseaux par exemple.

Certaines de ces glycoprotéines sont en outre porteuses de groupements sulfate très vraisemblablement fixés sur les oses neutres et/ou sur les osamines (Hedrick et al., 1974). Les glycoprotéines sulfatées sont relativement bien localisées au sein des gangues puisqu'on ne les rencontre que dans la gangue interne (et parfois, en plus faible quantité, dans la région interne de la gangue externe) (Humphries, 1970 ; Boisseau, 1973 ; Hédrick ef al., 1974 ; Yurewicz ef al.,-1975; Jégo, 1976).

Il n'y a d'ailleurs pas que les groupements sulfate qui soient distribués de manière hétérogène ; il faut en effet considérer que les gangues ovulaires d'Amphibiens sont constituées de plusieurs couches chimiquement très distinctes (Salthe, 1963 ; Humphries, 1966 ; Freeman, 1968 ; Shivers et James, $1970 \mathrm{~b}$; Pereda, 1970 ; Steinke et Benson, 1970 ; Boisseau et al., 1974). Ainsi chez le Pleurodèle où on peut isoler quatre fractions qui correspondent respectivement aux gangues interne, moyenne ef externe, les fractions interne et intéro-externe possèdent beaucoup plus d'acides aminés alcool (ser + thr) et de groupements sulfate que les deux autres fractions. La fraction interne est par ailleurs un peu plus riche en acides sialiques mais nettement plus pauvre en fucose que les autres fractions. S'il y a moins de fucose dans les produits de sécrétion qui constituent la gangue interne, cela provient en réalité de ce que ces produits ne sont pas « achevés» dans les conditions naturelles à cause d'un déficit en fucosyltransférase dans les cellules sécrétrices correspondantes. En effet, si on met ces produits en présence de fucosyltransférase exogène, ils sont capables de fixer des quantités très importantes de fucose supplémentaire ce qui n'est le cas d'aucun constituant des autres gangues. Il est à noter, enfin, qu'il n'est pas possible de fixer de galactose supplémentaire (même en présence de galactosyltransférase exogène) sur l'un quelconque des constituants des gangues (Jégo, 1978).

Les études immunochimiques et électrophorétiques indiquent que les gangues d'Amphibiens sont constituées d'un nombre assez limité de macromolécules différentes (une dizaine environ) (Yurewicz et al., 1975 ; Jégo, 1978). Les déterminations approchées de masse molaire par les techniques électrophorétiques révèlent des variations considérables dans la taille de ces glycoprotéines qui est voisine de 50000 daltons pour certaines, mais atteint plusieurs centaines de milliers et même quelques millions de daltons pour beaucoup d'autres. D'une manière générale, on peut considérer que, dans les conditions naturelles, les constituants des gangues se présentent en majoriłé sous forme de grosses molécules résultant éventuellement d'une polymérisation de monomères. Ainsi, par exemple les glycoprotéines les plus nombreuses de la gangue 
interne de Pleurodèle, dont la masse molaire mesurée en présence de dodécyl sulfate de sodium (agent dépolymérisant et dénaturant) est voisine de 50000 daltons, sont éluées en même temps que le bleu dextran ( 2 millions de daltons environ) sur une colonne de Sephadex G 100 dans des conditions non dénaturantes (Jégo, 1978).
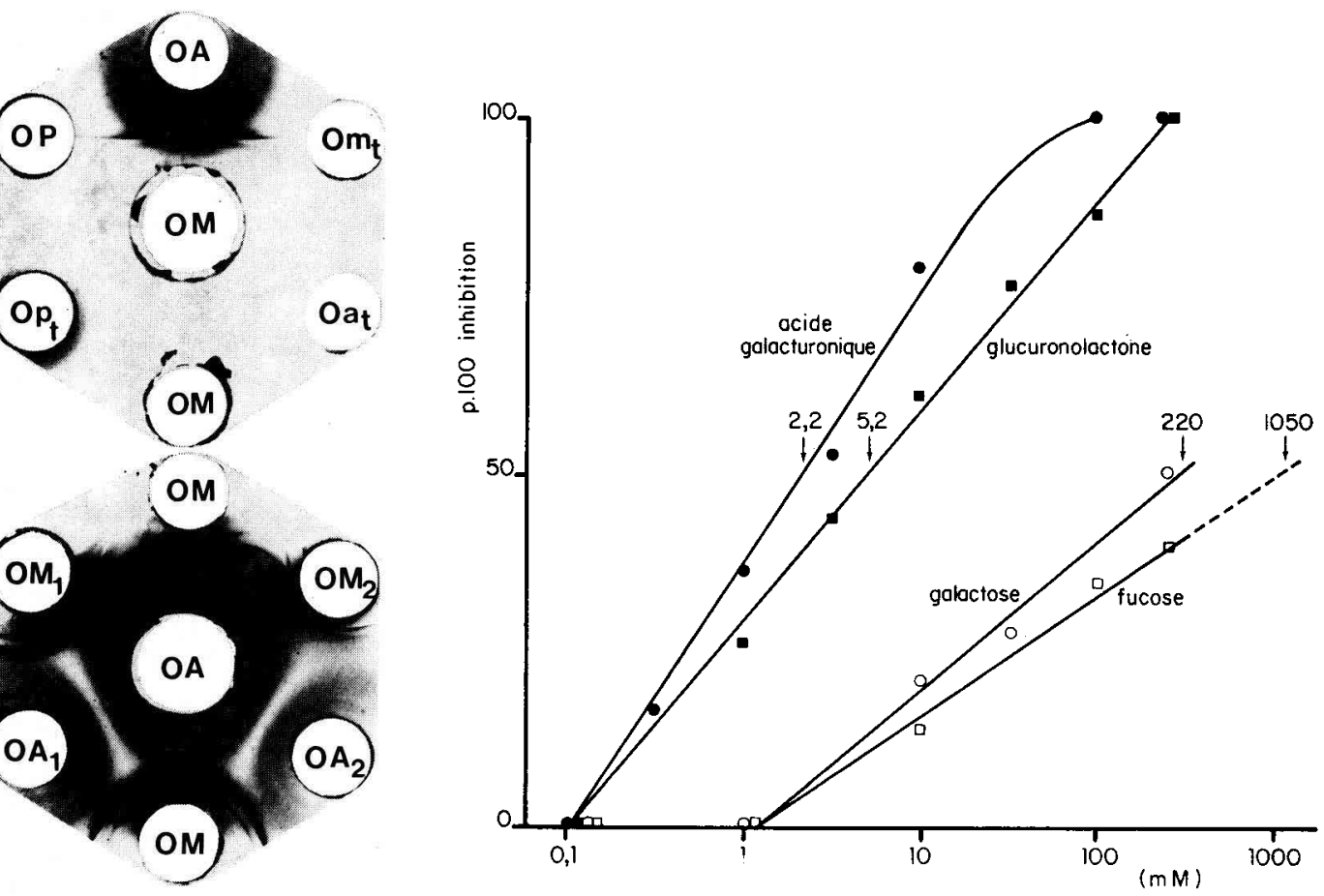

FIG. 2. - Réaction de précipitation intervenant enfre des produits de sécréfion de la région antérieure de l'oviducte $(O A)$ ef de la région $(O M)$ chez le Pleurodèle.

A gauche : Mise en évidence de la réaction de précipitation. Photographies de gels d'agarose au niveau desquels les surnageants d'homogénats des différents segments de l'oviducte (dans du tampon tris- $\mathrm{HCl} 0,1 \mathrm{M}, \mathrm{pH}: 7,5)$ sont confrontés. $\mathrm{OA}, \mathrm{OM}$ et $\mathrm{OP}$ désignent respectivement les segments antérieur, moyen et postérieur de l'oviducte d'un Pleurodèle adulte. $O a_{t}, 0 m_{t}$ et $0 p_{t}$ désigne ces mêmes segments chez un Pleurodèle immature dépourvu de produits de sécrétion. Afin d'essayer de mieux localiser les produits de sécrétion responsables de la réaction de précipitation, les segments antérieur et moyen ont parfois été divisés en deux parties : une partie antérieure $\left(O A_{1}\right.$ ou $\left.O M_{1}\right)$ et une partie postérieure $\left(O A_{2}\right.$ ou $\left.O M_{2}\right)$. Les conditions expérimentales sont celles utilisées couramment pour les expériences d'immunodiffusion sur gel d'agarose (Ouchterlony, 1949) à la différence près qu'il n'y a ici la présence d'aucun immun-sérum.

A droite : Effets inhibiteurs de quelques glucides sur la réaction de précipitation. Les mesures ont été réalisées par néphélométrie : lorsqu'on mélange dans un fube à essai les produits de sécrétion de $O A$ à ceux de $O M$, il apparaît un louche blanchâtre qui résulte de la réaction de précipitation. Par mesure directe de l'absorbance à $500 \mathrm{~nm}$, on peut ainsi quantifier l'intensité de cette réaction de précipitation et étudier, en particulier, l'effet inhibiteur de différents glucides sur la réaction obtenue après mélange, dans différents tubes à essais, de fractions aliquotes d'extraits de $O A$ et de $O M$. Les résultats sont exprimés en pourcentage d'inhibition par rapport au témoin. (Les concentrations des glucides capables de réduire de moitié l'intensité de la réaction sont portées sur la figure.) 


\section{Réaction de précipitation entre certaines protéines de l'oviducte du Pleurodèle ; isolement d'une protéine spécifique de l'oviducte antérieur}

Si on met en contact dans un tube à essai au ou sein d'un gel d'agarose les produits de sécrétion de l'oviducte antérieur (OA) et ceux de l'oviducte moyen (OM) du Pleurodèle, on constate qu'il apparaît un louche blanc dans le tube à essais ou deux arcs de précipifation dans le gel d'agarose qui témoignent de l'existence d'une réaction de précipitation intervenant entre certains produits de $O A$ et de $O M$ (fig. 2). Les produits qui interviennent dans cette réaction semblent être distribués de manière uniforme respectivement dans $O A$ ou dans $O M$ puisqu'on ne peut pas mettre en évidence une régionalisation au sein d'un même segment (fig. 2). La réaction de précipitation n'intervient qu'entre les produits de sécrétion puisque les oviductes d'animaux immatures ne réagissent pas (fig. 2). II faut noter cependant qu'il n'est plus possible d'obtenir une felle réaction entre les constituants des gangues ovulaires qui sont pourtant les produits de sécrétion de l'oviducte déposés autour de l'ovocyte. Cela tient très vraisemblablement au fait que la réaction a lieu dans les instants qui suivent l'excrétion naturelle des produits de telle sorte qu'on ne peut plus la reproduire plus tard ; même si toutes les molécules concernées n'ont pas toutes interagi entre elles, il est très probable par ailleurs que, par dénaturation elles perdent progressivement leurs propriétés particulières puisque celles-ci sont très dépendantes des conditions physicochimiques.

Cette réaction de précipitation est très sensible au $\mathrm{pH}$, à la force ionique ef à la concentration en manganèse du milieu ; elle est assez peu sensible à la température entre $0^{\circ} \mathrm{C}$ et $40^{\circ} \mathrm{C}$ mais, en revanche, elle est complètement modifiée lorsqu'on porte au préalable les produits de sécrétion de l'oviducte antérieur à $100^{\circ} \mathrm{C}$ pendant quelques minutes. Différents glucides inhibent la réaction de précipitation avec une efficacité qui varie selon leurs natures ; parmi les glucides que nous avons essayés (une trentaine) ce sont les acides uroniques qui exercent l'inhibition la plus importante (comme la réaction est sensible au $\mathrm{pH}$, nous utilisons la glucuronolactone de préférence à l'acide galacturonique dans les expériences courantes d'inhibition). L'allure des courbes présentées dans la figure 2 ainsi que l'observation d'une gradation de l'effet inhibiteur en fonction de la nature des glucides utilisés suggèrent une inhibition par compétition. Cette réaction de précipitation possède donc un certain nombre des caractéristiques des réactions de type lectine-oside.

Nous avons utilisé cette réaction de précipitation pour isoler par chromatographie d'affinité l'un des produits de sécrétion de l'oviducte antérieur. Dans un premier temps, l'ensemble des constituants de l'oviducte moyen est fixé de manière covalente sur du Sépharose $4 B$ activé au bromure de cyanogène. Après lavages et inactivation des sites du Sépharose n'ayant pas réagi, l'ensemble des constituants de l'oviducte antérieur est mis au contact de ce Sépharose particulier dans une colonne de chromatographie $(\mathrm{pH} 8,2)$. Celle-ci est lavée à plusieurs reprises avant d'y faire passer soit une solution de glucuronolactone $(0,5 \mathrm{M}, \mathrm{pH} 8,2)$ ce qui permet d'éluer une fraction $E_{1}$, soit une solution de $\mathrm{pH} 3,8$ (éluat $E_{2}$ ). La méthode utilisée permet, à partir d'un extrait donnant une vingtaine de bandes sur le protéinogramme (OA) d'isoler une protéine 
unique puisqu'il n'apparaît qu'une bande sur le protéinogramme (fig. 3). II s'agit d'ailleurs du produit de sécrétion le plus abondant de l'oviducte antérieur ; nous l'avons appelé « Spop ». Seul l'un des trois antigènes sécrétés par OA est présent dans $E_{2}$ ce qui semblerait indiquer que la technique utilisée aboutit à l'isolement d'une molécule immunologiquement pure (fig. 3). Si l'efficacité de la technique est excellente puisque de nombreuses protéines présentes dans l'oviducte antérieur ont été éliminées, il ne faut cependant pas totalement exclure la possibilité que l'éluat (et particulièrement $E_{2}$ obtenu après changement de $\mathrm{pH}$ ) renferme plusieurs molécules ayant le même déterminant antigénique et des propriétés électrophorétiques tellement voisines qu'on ne peut les séparer dans les conditions expérimentales utilisées. On remarque la destruction complète des sites antigéniques des produits de sécrétion de OA et notamment de la spop lors d'une dénaturation par la chaleur (fig. 3) ; ceci ne se produit pas pour les produits de sécrétion des autres segments de l'oviducte.
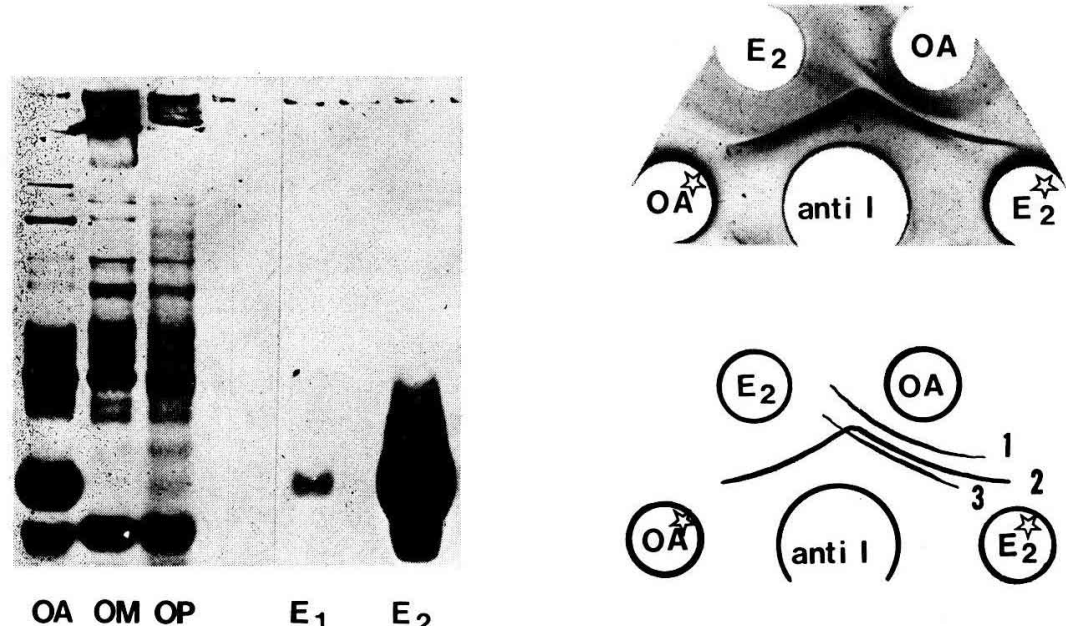

FIG. 3. - Isolement, par chromatographie d'affinité d'une protéine de l'oviducte antérieur du Pleurodèle Dans un premier temps, l'ensemble des constituants de l'oviducte moyen est fixé de manière covalente sur du Sépharose 4B activé au bromure de cyanogène. Après lavages et inactivation des sites du Sépharose n'ayant pas réagi, l'ensemble des constituants de l'oviducte antérieur est mis au contact de ce Sépharose particulier dans une colonne de chromatographie $(\mathrm{pH} \mathrm{8,2).} \mathrm{Celle-ci}$ est lavée à plusieurs reprises avant d'y faire passer soit une solution de glucuronolactone $(0,5 \mathrm{M}$, $\mathrm{pH} \mathrm{8,2)} \mathrm{ce} \mathrm{qui} \mathrm{permet} \mathrm{d'éluer} \mathrm{une} \mathrm{fraction} E_{1}$, soit une solution de $\mathrm{pH} 3,8$ (éluat $E_{2}$ ).

A gauche : Protéinogrammes obtenus oprès fractionnement par électrophorèse sur gel de polyacrylamide $(\mathrm{pH} \mathrm{8,0)}$ des protéines contenues dans les différents segments de l'oviducte $(O A, O M$, OP : segments antérieur, moyen et postérieur) et dans les éluats $E_{1}$ et $E_{2}$ (Les électrophorèses ont été réalisées en collaboration avec $\mathrm{H}$. Wroblewski.)

A droite : Efude immunochimique de l'éluat $E_{2}$. Les propriétés antigéniques de l'éluat $E_{2}$ et de la solution à partir de laquelle a été fait l'isolement (OA) sont comparées sur gel d'agarose (technique classique d'Ouchterlony, 1949) en utilisant un immunsérum de lapin dirigé contre la gangue ovulaire interne (c'est-à-dire les produits sécrétés puis excrétés par $O A$ ). $O A^{*}$ et $E_{2}{ }^{*}$ sont des extraits $O A$ et $E_{2}$ dénaturés pendant $10 \mathrm{mn}$ à $100^{\circ} \mathrm{C}$. 
Cette glycoprotéine est synthétisée sous l'action des hormones : absente chez les animaux immatures, on peut la faire apparaître après environ deux semaines de traitement par le $17 \beta$-œstradiol ; on ne la trouve alors que dans l'oviducte antérieur des animaux stimulés, dans des proportions sensiblement équivalentes à celles de l'adulte (Jégo ef Abalain, 1978).

\section{Rôles des gangues ovulaires}

De nombreux rôles sont dévolus aux gangues ovulaires, aussi bien au cours de la fécondation que durant la phase embryonnaire du développement. C'est grâce à leurs propriétés physicochimiques que l'œuf peut se fixer au substratum ou adhérer aux autres œufs. Leur consistance, coriace, élastique ou gluanle selon les cas peut jouer un important rôle dissuasif vis-à-vis de certains prédateurs ou parasites (Salthe et Mecham, 1974). Ces enveloppes peuvent, en outre, intervenir dans la réalisation précoce de la symétrisation de l'œuf (Beetschen, 1979), ainsi que dans la protection osmotique du jeune embryon (Salthe, 1965). Leur franchissement par la larve au moment de l'éclosion résulte, selon les cas, d'une action enzymatique ou mécanique (Noble, 1926 ; Katagiri, 1973). Mais, c'est sur leur importance dans le déroulement des stades précoces de la fécondation que nous voudrions attirer l'attention.

Remarquons en premier lieu que la consistance des gangues, surtout avant leur hydratation complète, en fait un milieu admirablement propice à la progression rectiligne des spermalozoïdes (Picheral, 1979). Une étude ultrastructurale (Picheral, 1977) montre que chez le pleurodèle, les spermatozoïdes se modifient au cours de la traversée des gangues. La coiffe acrosomienne a disparu lorsque les acrosomes pénètrent dans la gangue interne après avoir traversé la limite dense qui la sépare de la gangue moyenne. C'est donc à ce niveau que doivent intervenir la capacitation ef la réaction acrosomienne. Cette zone densifiée (fig. 1) pourrait résulter de l'affrontement, au cours du dépôt des gangues, des produits de sécrétion de l'oviducte antérieur et de l'oviducte moyen.

Si l'on met en contact des spermatozoïdes de pleurodèle avec l'une ou l'autre des gangues isolées ou avec des extraits de gangues, les spermatozoïdes ne subissent pas la réaction acrosomienne et ils restent inaptes à féconder les ovocytes dégangués. La même expérience, réalisée chez divers Anoures, aboutit au contraire à des fécondations (Vorps et Elinson, 1979). On a même pu isoler (Oliphant ef Hédrick, 1971) des «facteurs de capacitation», habituellement glycoprotéiques dont la masse molaire varie selon les espèces. La capacitation est donc un phénomène apparemment plus complexe chez les Urodèles que chez les Anoures. Elle comporte peut-être des interactions séquentielles (chimiques ou/et physiques) de différentes molécules avec les spermatozoïdes. En l'état actuel des connaissances, on ne peut que formuler des hypothèses à cet égard.

Parmi les constituants des gangues ayant des propriétés physicochimiques particulières et susceptibles d'intervenir dans ces interactions, on peut, chez le pleurodèle, mettre l'accent sur trois catégories de molécules.

Il faut noter, en premier lieu, l'existence dans la gangue moyenne d'une protéine nettement basique puisqu'à $\mathrm{pH} 8,5$ et même 9,0 , elle est chargée positivement contraire- 
ment à tous les autres constituants des gangues. Cette propriété pourrait être l'une des causes de la grande cohésion des consituants de la gangue moyenne ; cette molécule basique pourrait en outre interagir directement avec des molécules de la membrane du spermatozoïde chargées négativement, des acides sialiques par exemple.

Les glycoprotéines de la gangue interne renferment, par ailleurs, des quantités de fucose nettement moins importantes que les constituants des autres gangues. Cette pauvreté en fucose est codée génétiquement puisque nous avons vu qu'elle résulte d'un déficit en fucosyltransférase des cellules sécrétrices correspondantes ; il faui noter par ailleurs que la fucosyltransférase, lorsqu'elle est présente, est synthétisée sous l'effet des hormones contrairement à la galactosyltransférase par exemple (Jégo, 1977). La présence ou l'absence du fucose dans les produits de sécrétion de l'oviducte du pleurodèle semble ainsi être très efficacement contrôlée. On peut imaginer que des liaisons stables apparaissent entre des ectofucosyltransférases portées par la membrane plasmique du spermatozoïde d'une part et les accepteurs de fucose de la gangue interne d'autre part. En l'absence de GDP-fucose (rare en milieu extracellulaire), le complexe qui pourrait ainsi être formé serait en effet relativement stable.

Il faut rappeler enfin la réaction de précipitation de type lectine-oside décrite plus haut. L'hypothèse d'une telle réaction intervenant entre la « spop » et des glucides du revêtement externe de la membrane plasmique du spermatozoïde n'est pas à exclure. Cette réaction ne pourrait se produire que pendant un temps très bref, puisque les molécules de «Spop » réagissent avec les molécules de la gangue moyenne, formant ainsi la zone densifiée qui sépare les deux gangues.

Une autre éfape importante pour la réussite de la fécondation est le blocage à la polyspermie. Là encore, Anoures et Urodèles ont mis en place des mécanismes différents même s'ils comportent quelques analogies.

L'œuf des Anoures est normalement monospermique. Cette monospermie semble s'établir en deux temps (Gross et Elinson, 1978). Une phase très précoce, au cours de laquelle les spermatozoïdes seraient, comme chez les Echinodermes, immobilisés à la suite d'une variation du potentiel de membrane de l'œuf, elle-même déclenchée immédiatement par la pénétration du spermatozoïde fécondant. Une phase un peu plus fardive correspond à la libération des granules corticaux (Wyrick ef al., 1974) dont le contenu interagit avec un ou des produits de la gangue interne. Le produit de la réaction se dépose à l'extérieur de l'enveloppe vitelline ef semble constituer une barrière infranchissable aux spermatozoïdes (Grey et al., 1976).

Contrairement à celui des Anoures, l'œuf des Urodèles est normalement polyspermique. On ignore encore s'il existe chez eux un mécanisme précoce de limitation de la polyspermie. Ce n'esi pas évident étant donné le mode d'insémination intracloacale qui caractérise les Urodèles supérieurs (Boisseau ef Joly, 1975). Toutefois, on constate, au cours de fécondations artificielles qu'une grande majorité des spermatozoīdes mis au contact des œufs restent bloqués au niveau de la zone densifiée (fig. 1) qui leur devient de moins en moins perméable au fur et à mesure que le temps passe. On aboutit finalement à l'installation d'une barrière aux spermatozoïdes vingt minutes environ après la ponte des œufs.

En conclusion, la connaissance de l'organisation des gangues qui enveloppent l'œuf des Amphibiens et de leur constitution chimique a progressé durant les dernières 
années. Elle est l'une des conditions essentielles pour parvenir à une analyse satisfaisante des mécanismes cellulaires impliqués dans les phases précoces de la fécondation.

Présenté au Colloque D.G.R.S.T. de Port Bail,

27 février-1er mars 1979.

Accepté en novembre 1979.

Remerciements. - Les auteurs remercient Mlle S. de Sallier-Dupin pour la dactylographie du manuscrit.

\section{Références}

APLINGTON H. W., 1957. The insemination of body cavity and oviducal eggs of Amphibia. Ohio J. Sci., 57, $91-99$.

BATAILLON E., 1919. Analyse de l'activation par la technique des œuís nus et la polyspermie expérimentale chez les Batraciens. Ann. Sci. nat. zool., 10, 1-38.

BAULIEU E. E., GODEAU F., SCHORDERET M., SCHORDERET-SLATKINE S., 1978. Steroid-induced meiotic division in Xenopus laevis oocytes : surface and calcium. Nature, 275, 593-598.

BEETSCHEN J. C., 1979. Recherches expérimentales sur la symétrisation de l'oocyte et de l'œuf d'Axolotl : facteurs conditionnant l'apparition précoce du croissant gris à la suite d'un choc thermique. C. R. Acad. Sci. Paris, sér. D, 288, 643-646.

BOISSEAU C., 1973. Données autoradiographiques sur l'incorporation au sulfate de sodium radioactif dans l'oviducte de Pleurodeles waltlii Michah. (Amphibien, Urodèle, Salamandridé). C. $R$. Soc. Biol., 167, 746-750.

BOISSEAU C., JEGO P., JOLY J., PICHERAL B., 1974. Organisation et caractérisation histochimique des gangues ovulaires sécrétées par l'oviducte de Pleurodeles walllii Michah (Amphibien, Urodèle, Salamandridé). C. R. Soc. Biol., 168, 1102-1107.

BOISSEAU C., JOLY J., 1975. Transport and survival of spermatozoa in female Amphibia, 94-104. In HAFEZ E. ef THIBAULT Ch. Karger, Basel.

BOLOGNANI L., BOLOGNANI-FANTIN A. M., LUSIGNANI R., TONTA L., 1966. Presence of sialopolysaccharidic components in egg gelatinous mantle of Rana latastei and Bufo vulgaris. Experientia, 22, 601-603.

DE MARTINEZ N. R., MENDEZ B. A., OLAVARRIA J. M., 1975. Topographical distribution of sialic acids and related enzymes in toad oviduct. Comp. Biochem. Physiol., 50B, 603-607.

EPEL D., 1978. Le programme de la fécondation. Pour la science, $n^{\circ} 3,34-45$.

FOLKES B. F., GRANT R. A., JONES J. K. N., 1950. Frog-spawn mucin. J. Chem. Soc., 440, 2136.

FREEMAN S. B., 1968. A study of the jelly envelopes surrounding the egg of the amphibian, Xenopus laevis. Biol. Bull., Woods Hole, 135, 501-513.

GREY R. D., WORKING P. K., HEDRICK J. L., 1976. Evidence that the fertilization envelope blocks sperm entry in eggs of Xenopus laevis : Interaction of sperm with isolated envelopes. Devel Biol., 54, 52-60.

GROBSTEIN C., 1979. La fécondation externe chez l'homme. Pour la Science, $n^{\circ}$ 22, 67-78.

GROSS N. L., ELINSON R. P., 1978. Rapid block to polyspermy in frog mediated by changes in the membrane potential. Am. Zool., 18, 413.

HEDRICK J. L., SMITH A. J., YUREWICZ E. C., OLIPHANT G., WOLF D. P., 1974. The incorporation and fate of $\left[{ }^{35} \mathrm{~S}\right]-$ sulfate in the jelly coat of Xenopus laevis eggs. Biol. Reprod., 11, 534-542.

HUMPHRIES A. A., 1966. Observations on the deposition, structure and cytochemistry of the jelly envelopes of the egg of the newt, Triturus viridescens. Develop. Biol., 13, 214-230.

HUMPHRIES A. A., 1970 . Incorporation of $\left[{ }^{35}\right.$ S ] sulfate into the oviducts and egg jelly of the newt, Notophtalmus viridescens. Exp. Cell Res., 59, 157-161.

JEGO P., 1974. Composition en glucides des différents segments de l'oviducte et des gangues ovulaires chez Pleurodeles waltlii Michach. (Amphibien, Urodèle). Comp. Bioch. Physiol., 47, 435-446.

JEGO P., 1976. Analyse des protides des gangues ovulaires de Pleurodeles waltili Michah (Amphibien, Urodèle). Ann. Biol. onim. Bioch. Biophys., 16, 13-24.

JEGO P., 1977. Action différentielle de l'œestradiol sur les activités de la fucosyltransférase et de la galactosyltransférase de l'oviducte du triton pleurodèle. Gen. comp. Endocr., 31, 475-481. 
JEGO P., 1978. Analyse chimique et immunochimique des gangues ovulaires sécrétées par l'oviducte du pleurodèle ; effets de l'astradiol sur les activités enzymatiques des cellules sécrétrices. Th. Ełat, Univ. Rennes, série C, no de série 80.

JEGO P., ABALAIN J. H., 1978. Isolement, par chromatographie d'affinité, d'une protéine spécifique apparaissant dans l'oviducte du Pleurodèle immature sous l'effet de l'œstradiol. Biol. Cell., 31, 311-314.

JOLY J., 1980. Les gangues ovulaires et leurs rôles. Traité de Zoologie, In P. P. GRASSÉ. Tome XIV, Amphibiens I. Masson ed. Paris (sous presse).

KATAGIRI C., 1966. Fertilization of dejellied uterine toad eggs in various experimental conditions. Embryologica, 9, 159-169.

KATAGIRI C., 1973a. Chemical analysis of toad eggs-jelly in relation to its "sperm capacitating 》 activity. Develop. Growth Different., 15, 81-92.

KATAGIRI C., 1973b. A method for assaying the jelly-digesting activity of the hatching enzyme from frog embryos. J. Fac. Sci. Hokkaido Univ., ser. VI, Zool., 18, 461-468.

LEE P., 1967. Studies of frog oviducal jelly secretion. I. Chemical analysis of secretory product. Exp. Zool., 166, 99-106.

METZ C. B., 1967. Gamete surface components and their role in fertilization. In METZ C. B. MONROY A., "Fertilization : Comparative morphology, biochemistry and immunology ", Acad. Press, New York, vol. 1, 163-236.

MINGANTI A., D'ANNA T., 1957. Ricerche sulla mucina ovulare di Triton cristatus. Ric. sci., Anno, 27, 3052-3054.

MINGANTI A., D'ANNA T., 1958. Sulla composizione della mucina ovulare di Discoglossus pictus. Ric. Sci. Annc, 28, 2090-2093.

NOBLE G. K., 1926. The hatching process in Alytes, Eleutherodactylus and other Amphibians. Amer. Mus. Novit., 229, 1-7.

OLIPHANT G., HEDRICK J. L., 1971. Isolation and physicochemical characterization of a sperm capacitation factor from the jelly coat of Xenopus laevis eggs. Fed. Proc., 30, 1280.

OUCHTERLONY O., 1949. Antigen-antibody reactions. Acto pothol, microbiol. scand., 26, 507-515.

PEREDA J., 1970. Etude histochimique de la distribution des sialomucines dans l'oviducte et les gangues muqueuses des ovocytes de Rana pipiens. Comportement dans l'eau des différentes gangues. J. Embryol. exp. Morph., 24, 1-12.

PICHERAL B., 1977. La fécondation chez le triton Pleurodèle. I. La traversée des enveloppes de l'ceuf par les spermatozoïdes. J. Uitrastruct. Res., 60, 106-120.

PICHERAL B., 1979. Structural, comparafive and functional aspects of spermatozoa in Urodeles 267-287. In D. W. FAWCETT, J. M. BEDFORD, The spermatozoon Urban and Schwarzenberg, Baltimore.

SALTHE S., 1963. The egg capsules in the amphibia. J. Morphol., 113,161-171.

SALTHE S. N., 1965. Increase in volume of the perivitelline chamber during development of Rana pipiens Schreber. Physiol. Zool., 38, 80-98.

SALTHE S. N., MECHAM, J. S., 1974. Reproductive and courtship patterns, 309-521. In LOFTS B., Physiology of the Amphibia, vol. 11, Acad. Press.

SHIVERS C. A., JAMES J. M., 1970a. Capacitation of frog sperm. Nature, 227, 183-184.

SHIVERS C. A., JAMES J. M., 1970b. Morphology and histochemistry of the oviduct and egg-jelly layers in the frog, Rana pipiens. Anat. Rec., 166, 541-556.

STEINKE J. H., BENSON D. G., 1970. The structure and polysaccharide cytochemistry of the jelly envelopes of the egg of the frog, Rana pipiens. J. Morphol., 130, 57-66.

SPALLANZANI L., 1785. Expériences pour servir à l'histoire de la génération des animaux et des plantes. Chirol (Genève), 413 p.

THIBAULT C., 1975. La fécondation chez les Mammifères. Masson ed.

THIBAULT C., 1976. La fécondation. La Recherche, 7, 411-420.

VORPS M. M., ELINSON R. P., 1979. Extraction of oviducal materials important in the fertilization of Rana pipiens eggs. Biol. Reprod., 20, suppl. no 1, $91 \mathrm{~A}$.

WYRICK R. E., NISHIHARA T., HEDRICK J. L., 1974. Agglutination of jelly coat and cortical granule components and the block to polyspermy in the Amphibian Xenopus laevis. Proc. nat. Acad. Sci., U. S. A., 71, 2067-2071.

YUREWICZ E. C., OLIPHANT G., HEDRICK J. L., 1975. The macromolecular composition of Xenopus loevis egg jelly coał. Biochemistry, 14, 3101-3107. 